Huysse: Ueber den mikrochemischen Nachweis des Kaliums ete.

\title{
Ueber den mikrochemischen Nachweis des Kaliums, Rubidiums, Caesiums, Indiums und von Thiosulfaten. \\ Yon
}

A. C. Huysse, Stabsapotheker.

(Mit einer lithographirten Tafel 1).

In dieser Zeitschrift 36, 512 findet man eine Methode zur Prüfung auf Kalium mittelst Natrium-Wismuththiosulfats erwähnt, welche, wie sich bei näherer Prüfung ergab, auch zum mikrochemischen Nachweis der Metalle Kalium, Rubidium und Caesium geeignet ist. Das Reagens zu diesem $Z$ weck wird am schnellsten dargestellt, indem man ein wenig basisches Wismuthnitrat auf einem Uhrglase in möglichst wenig Salzsäure löst und Wasser zufügt, bis sich ein dicker, weisser Niederschlag absetzt, welcher durch Versetzung mit Natriumthiosulfat wieder in Lösung gebracht wird. Man achte darauf, nicht mehr Thiosulfat anzuwenden, als zur Auflösung des Niederschlages erforderlich ist. Eine derartige Lösung ist gelb gefärbt. Sie wird mit starkem Weingeist gemischt, bis eine dauernde Trübung hervorgerufen wird. Wenig Wasser lässt dieselbe sofort wieder verschwinden, und das Reagens ist fertig. Haltbar ist es nicht, denn es erleidet bald Zersetzung, indem sich Schwefelwismuth bildet, und ist jedesmal frisch zu bereiten.

Die auf Kalium zu prüfende Flüssigkeit kann zuvor auf dem Objectträger bis zur Trockne verdunstet oder mit Weingeist bis auf dieselbe Stärke wie das Reagens versetzt werden, um zu verhüten, dass durch zu starken Weingeist eine Trübung hervorgerufen wird, oder um dem Ausbleiben der Reaction durch zu starke Verdünnung des Alkobols vorzubeugen.

Ist Kalium zugegen, dann entstehen sofort schöne, gelbgrüne Nädelchen, dem monoklinen Systeme angehörend, wenn ein Tröpfchen des Reagens zugesetzt wird (Fig. 1 auf Taf. I, Kalium-Wismuththiosulfat). Diese Reaction ist recht empfindlich; mit 0,002 $\mathrm{mg} \mathrm{KNO}_{3}$ kamen noch Krystalle zum Vorschein, und 0,010 $\mathrm{mg}$ verursachte starke Fällung.

Der Vortheil dieses Verfahrens liegt darin, dass Ammonsalze und die Salze anderer Metalle, Rubidium und Caesium ausgenommen, keinen Niederschlag erzeugen und nichts an der Reaction abändern.

1) Die Abbildungen sind aus freier Hand gezeichnet; die Vergrösserung. ist eine 240 fache. 
Rubidium- und Caesiumsalze veranlassen ebenfalls die Entstehung gelbgrüner Nädelchen, welche denen des Kaliumsalzes völlig ähnlich sind. In verdünntem Weingeist sind sie weniger löslich und erscheinen öfter zu Bündeln gruppirt. (Taf. I, Fig. 2 a Rubidium- und Fig. 2 b Caesium - Wismuththiosulfat.)

Salze der alkalischen Erden fällen weiss, aber diese Niederschläge sind für die Mikrochemie von keinem Werth. Oben genannte Reaction ist also nur dann entscheidend für Kalium, falls weder Rubidium noch Caesium zugegen ist.

\section{Zum mikrochemischen Nachweis des Indiums.}

a) Caesiumchlorid bewirkt in ziemlich verdünnten Lösungen von Indiumsulfat, welche ein wénig freie Schwefelsäure enthalten, die Entstehung farbloser, gut ausgebildeter Octaëder von Caesium-Indiumsulfat oder Caesium-Indiumalaun.

Ein Tropfen der Flüssigkeit, welche auf Indium geprüft werden soll, wird mit einem Tröpfcheń Schwefelsäure auf dem Objectglase abgedampft, der Rückstand in wenig Wasser gelöst, und ein Kryställchen Caesiumchlorid zugesetzt. (Taf. I, Fig. 5, Caesium-Indiumalaun.)

b) Fällung mit Ammoniumfluorid als Ammoniumfluoindat.

Ammoniumfluorid fällt Indinmsalze in derselben Weise wie Aluminiumsalze. Wird Ammoniumfluorid im Ueberschuss zugesetzt, so bilden sich eben so gut ausgebildete Octaëder, wie wenn es sich um. Aluminium handelte. (Fig. 6, Taf. I, Ammoniumfluoindat.)

c) Fällung mit Mercurisulfocyanat. Schon in beträchtlicher Verdünnung fällt eine Lösung von Ammonium-Mercurisulfocyanat aus Indiumlösungen farblose Krystalle, welche denen des Zink- oder CadmiumMercurisulfocyanats nicht ganz ähnlich sind. (Fig. 7, Taf. I, IndiumMercurisulfocyanat.) Sind andere mit Mercurisulfocyanat fällbare Metalle, wie Zink, Cadmium, Kupfer oder Kobalt, zugegen, so entstehen Mischkrystalle.

d) Oxalsäure und oxalsaures Ammon fällen Indiumlösungen. Die Reaction ist recht empfindlich. Der Niederschlag besteht aus farblosen Krystallen, welche denen des Zinkoxalats einigermaassen gleichen. Ist viel freie Säure zugegen, so wird die Fällung dadurch bedentend verzögert. Zusatz von Ammoniak beschleunigt die Reaction. Zum Unterschiede vom Zinkoxalat ist das Indiumoxalat in Ammoniak vicht löslich. (Fig. 8, Taf. I, Indiumoxalat.) Prüft man Indiumlösungen mit anderen 
gebräuchlichen Reagentien, so erhält man bisweilen Niederschläge, zum Beispiel mit Kali und Natron, Ammoniak, kohlensauren Alkalien und Ferrocyankalium, aber sie sind von geringem Werth für dieses Verfahren.

Die Reactionen a und $b$ sind nur dann entscheidend für Indium, wenn die Anwesenheit von Aluminium, und für b auch die des Eisens, ausgeschlossen ist, und die übrigen $\mathrm{c}$ and $\mathrm{d}$ erfordern wenigstens die Entfernung der Metalle Zink, Cadmium, Kupfer, Kobait und Eisen.

Die Methode K. J. Bayer's ${ }^{1}$ ) zur Darstellung des Indiums aus dem Schlamme des Freiberger Zinks ist auch vortheilhaft, wenn es gilt, das Indium aus Lösungen gemengter Verbindungen zu isoliren.

Am Schluss dieses Verfahrens erhält man ziemlich reines schwefligsaures Indium, nur verunreinigt mit Metallen, welche den Reactionen nicht schädlich sind.

\section{Zum mikrochemischen Nachweis von Thiosulfaten.}

Auf den Umstand, dass Thallium- und Bleisalze Thiosulfatlösungen krystallinisch fällen, lässt sich vielleicht eine Methode zum mikrochemischen Nachweis von Thiosulfaten gründen. Ein Körnchen Thallonitrat, in eine Lösung von Natriumthiosulfat gebracht, veranlasst Fällung farbloser Krystalle, vielleicht herrührend von Thallothiosulfat. Ein Ueberschuss von Thiosulfat hat die Entstehung eines Doppelthiosulfats von Natrium und Thallium zur Folge, das ja bedeutend löslicher ist und bisweilen zur Krystallisation gelangt. Die Kreuze und tafelförmigen Krystalle ändern sich dann zu Büscheln langer, dünner, seideglänzender Nädelchen um.

Sulfate, Nitrate und Acetate ändern nichts an der Reaction, nur die Chloride, Bromide und Jodide haben einen störenden Einfuss.

Mit $0,01 \mathrm{mg}$ Natriumthiosulfat erhielt ich noch gut ausgebildete Krystalle. (Fig. 3, Taf. I, Thallothiosulfat.) Die Reaction mit Bleiacetat ist weniger geeignet, denn der Niederschlag in ziemlich verdünnter Lösung von Thiosulfat ist feinkörnig, und in stärkerer bekommt man sehr leicht verschiedene Doppelsalze. Es entstehen Rauten mit spitzem Winkel von $67^{\circ}$ and auch Nadeln, zu Bündeln angehäuft. (Fig. 4, Taf. I.)

Die Fällung mit Thallonitrat verdient also den Vorzug. Gorinchem, September 1899.

1) Ann. d. Chem. u. Pharm. 158, S. 372; diese Zeitschrift 11, 77. 